\title{
Independent occurrence of de novo HSPD1 and HIP1 variants in brothers with different neurological disorders - leukodystrophy and autism
}

\author{
Toshiyuki Yamamoto (1) ${ }^{1,2}$, Keiko Yamamoto-Shimojima ${ }^{1,2}$, Yuki Ueda ${ }^{3}$, Katsumi Imai ${ }^{3}$, Yukitoshi Takahashi ${ }^{3}$, \\ Eri Imagawa ${ }^{4}$, Noriko Miyake ${ }^{4}$ and Naomichi Matsumoto ${ }^{4}$
}

\begin{abstract}
Consecutive occurrence of de novo variants in the same family is an extremely rare phenomenon. Two siblings, a younger brother with hypomyelinating leukodystrophy and an elder brother with severe intellectual disability and autistic features, had independent de novo variants of HSPD1 c.139T > G (p.Leu47Val) and HIP1 c.1393G > A (p. Glu465Lys), respectively. These novel variants were predicted to be pathogenic. Both patients also had a known MECP2 variant, c.499C > T (p.Arg167Trp).
\end{abstract}

Hypomyelinating leukodystrophies (HLDs) are caused by congenital dysfunctions of the oligodendroglia and/or astrocytes ${ }^{1}$. Patients with HLDs show motor developmental delay and other neurological symptoms, including intellectual disability (ID), nystagmus, tremor, and epilepsy. Brain magnetic resonance imaging (MRI) demonstrates -T2-high intensity in the white matter ${ }^{2}$. OMIM (https://www.omim.org/) registers 13 subgroups of HLDs (Supplemental_Table_S1) ${ }^{3,4}$. Pelizaeus-Merzbacher disease (PMD; MIM\#31208) is the most common HLD in male patients and is classified as HLD1. Because the proteolipid protein 1 gene (PLP1) responsible for PMD is located on the $\mathrm{X}$-chromosome, $\mathrm{PMD}$ is an $\mathrm{X}$-linked recessive disease.

Among HLDs, only HLD6 (MIM\#612438) is related to autosomal dominant traits. Most patients with HLD6 have de novo variants in the tubulin beta- $4 \mathrm{~A}$ gene $(T U B B 4 A)^{5}$, whereas TUBB4A is also related to autosomal

\footnotetext{
Correspondence: Toshiyuki Yamamoto (yamamoto.toshiyuki@twmu.ac.jp) ${ }^{1}$ Institute of Medical Genetics, Tokyo Women's Medical University, Tokyo, Japan ${ }^{2}$ Tokyo Women's Medical University Institute for Integrated Medical Sciences, Tokyo, Japan
}

Full list of author information is available at the end of the article. dominant torsion dystonia 4 (DYT4; MIM\#128101). In families with DYT4, TUBB4A variants are inherited as autosomal dominant traits. Thus, HLD6 and DYT4 are recognized as allelic disorders. The clinical difference between HLD6 and DYT4 is unclear, and there is an intermediate pattern ${ }^{5}$. Similarly, the $60-\mathrm{kDa}$ heat shock protein 1 gene (HSPD1), responsible for HLD4 $(\mathrm{MIM} \# 612233)^{6}$, is associated with spastic paraplegia 13 (SPG13; MIM\#605280) ${ }^{7}$, suggesting that HLD4 and SPG13 are also allelic disorders.

Here, we report on a de novo heterozygous HSPD1 variant in a sporadic patient with HLD4 but not SPG13 (patient 1, Supplemental Information) in association with a de novo variant in the huntingtininteracting protein 1 gene (HIP1), which was concurrently identified in his elder brother (patient 2, Supplemental Information).

In accordance with the declaration of Helsinki, this study was approved by the ethics committee of Tokyo Women's Medical University. After receiving written informed consent from the family, we obtained blood samples from siblings and their parents. Genomic DNA was extracted for sequence analysis and used for genetic diagnosis. 
Because PMD derived from duplications or singlenucleotide alterations in the PLP1 region is the major type of HLD, PLP1 analysis by microarray-based comparative genomic hybridization and Sanger sequencing was performed as the first tier $^{8}$, and these confirmed no abnormalities. Whole-exome sequencing (WES) was performed for both parents and siblings (patients 1 and 2) as described ${ }^{9}$. Synonymous variants, variants with more than $1 \%$ global population frequency, and variants registered in the Human Genetic Variation Database ${ }^{10}$ were filtered. Variants with de novo origin or those showing Mendelian inheritance patterns, associated with autosomal dominant or recessive traits, were manually selected. Pathogenic genomic copy-number variations (CNVs) were not identified by the eXome Hidden Markov Model using the data extracted through WES ${ }^{11}$.

Novel variants, NM_002156.4(HSPD1):c.139T > G (p.Leu47Val) and NM_005338.6(HIP1):c.1393G > A (p.Glu465Lys), were identified in patients 1 and 2 , respectively. These variants were not registered in the 1KGP, ExAC (http://exac.broadinstitute.org/), ESP6500 (http://evs.gs.washington.edu/EVS/), or iJGVD database (Supplemental Table S3, 4) ${ }^{12,13}$. At the same position on HIP1 c.1393G > A, there is a known SNP, rs782598703G $>$ C (p.Glu465Gln) (location chr7:75187292), with a minor allele frequency of $0.001 \%(1 / 121394)$. Both patients and their mother carried NM_004992.3(MECP2):c.499C > T (p.Arg167Trp), registered as rs61748420 (Supplemental Table S2, 5), indicating X-linked recessive inheritance. There was no other possible candidate variant in the genes related to the clinical features in association with Mendelian inheritance, including autosomal-recessive traits. The results are summarized in Table 1, together with clinical features. All variants were confirmed by Sanger sequencing (Fig._1c), and damaging scores predicted through wANNOVAR (http://wannovar.wglab.org/ ) are listed in Supplemental_Table_S2. Most of the scores, including CADD, suggested pathogenicity. The results of wANNOVAR analysis for all previously reported HSPD1 variants are compared in Supplemental_Table_S3, suggesting no significant difference from the present variant. The amino acid sequences affected by all variants discussed in this study are conserved among species (Supplemental_Figure_S1).

Protein secondary structures were predicted through web-based software provided by PRABI-Lyon-Gerland (The Institute of Biology and Chemistry of Proteins, Lyon, France; https://prabi.ibcp.fr/htm/site/web/home). A marked change was shown by HSPD1 p.Leu47Val but not by HIP1 p.Glu465Lys (Supplemental Figure S2,3).

Patient 1 showed progressive paraplegia due to unknown leukodystrophy (Fig._1a, b), and HLD4 was considered as a clinical diagnosis rather than SPG13. Although bi-allelic HSPD1 involvement was expected ${ }^{6}$,
Table 1 Summary of the clinical features and gene variants

\begin{tabular}{|c|c|c|}
\hline & Patient 1 & Patient 2 \\
\hline Gender & Male & Male \\
\hline Age & 8 years & 12 years \\
\hline \multicolumn{3}{|l|}{ Stature at birth } \\
\hline Weight (g) & 2368 & 2470 \\
\hline OFC (cm) & 29 & 33 \\
\hline \multicolumn{3}{|l|}{ Stature at last examination } \\
\hline Height & {$[-4.0 S D]$} & {$[-2.8 \mathrm{SD}]$} \\
\hline Weight & {$[-2.3 S D]$} & {$[-2.1 \mathrm{SD}]$} \\
\hline \multicolumn{2}{|l|}{ delay } & Moderate \\
\hline Standing/walking & 12 mo. & 20 mo. \\
\hline Febrile seizures & + & + \\
\hline Intractable seizures & + & - \\
\hline Pyramidal sign & + & - \\
\hline Dysarthria & + & - \\
\hline Cerebellar signs & + & - \\
\hline Developmental deterioration & + & - \\
\hline Abnormal MRI findings & + & - \\
\hline Cognitive impairment & - & + \\
\hline Intellectual disability & - & + \\
\hline Severe autistic features & - & + \\
\hline \multicolumn{3}{|l|}{ Gene variants } \\
\hline HSPD1 p.Leu47Val & + & - \\
\hline HIP1 p.Glu465Lys & - & + \\
\hline MECP2 p.Arg167Trp & + & + \\
\hline
\end{tabular}

OFC occipito-frontal circumference, $S D$ standard deviation, mo months

only a hemi-allelic involvement was confirmed. This may have reflected the existence of an unidentified variant in the non-coding region of HSPD1, because pathogenic $\mathrm{CNVs}$ were not observed. Another possible explanation is that HLD4 in this patient was caused by hemi-allelic involvement of HSPD1.

An example of a gene related to both autosomalrecessive and dominant traits is the hepatic and glial cell adhesion molecule gene $(H E P A C A M)^{14}$. Megalencephalic leukoencephalopathy with subcortical cysts $2 \mathrm{~A}$ and $\mathrm{B}$ (MLC2A MIM\#613925; MLC2B MIM\#613926) is related to $H E P A C A M$ variants in recessive and dominant patterns, respectively. Although HLD4 and SPG13 are considered to be inherited through different modes, with autosomal-recessive and dominant traits, the main clinical 

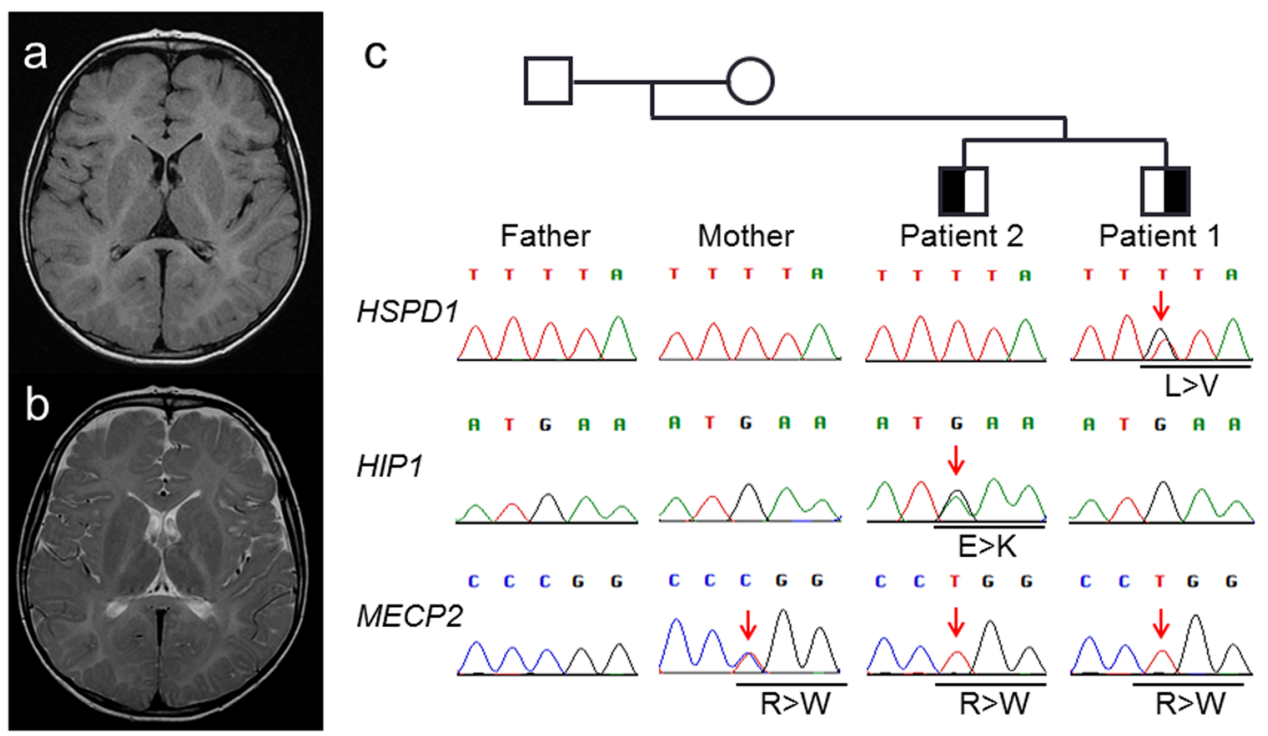

Fig. 1 Results of brain MRI and molecular investigations. T1- (a) and T2-weighted axial images (b) of patient 1 examined at 4 years old. A -T2high-signal is noted in the white matter, indicating hypomyelination. c Electropherograms of Sanger sequencing depicted in the family tree. Variants in HSPD1 and HIP1 are identified only in patient 1 and patient 2, respectively. Alternatively, the MECP2 variant is identified in both patients as hemizygous and in their mother as heterozygous, indicating an X-linked recessive variant

features are commonly derived from pyramidal tract involvement owing to the dysfunction of myelin. Therefore, HLD4 and SPG13 may be involved in the same clinical spectrum, and the HLD4 in this patient may have been derived from hemi-allelic involvement in HSPD1, as in SPG13.

Only four HSPD1 variants, pVal98Ile ${ }^{7}$, p.Gln $461 \mathrm{Glu}^{15}$, and p.Gly563Ala ${ }^{16}$, have been reported as pathogenic variants for the autosomal dominant trait of SPG13, and only one homozygous variant, p.Asp29Gly, has been reported for the autosomal-recessive trait of HLD4 ${ }^{6}$, indicating that bi-allelic involvement is rare. In our in silico analysis of the novel variant of p.Leu47Val, a marked conformational change of HSPD1 protein was predicted. Thus, a dominant negative effect of p.Leu47Val was suspected, and it may have been responsible for the autosomal dominant trait of HLD4.

In contrast to patient 1 , patient 2 did not show any abnormal findings on brain MRI. This is reasonable because patient 2 did not have the HSPD 1 variant. Rather, he showed severe neuropsychiatric features, including ID, severe autistic features, and intractable epilepsy. WES identified a de novo HIP1 (the huntingtin-interacting protein 1 gene) variant. HIP1 is located on the neighboring region of the William syndrome critical region in $7 \mathrm{q} 11.23$, and atypically large deletions in $7 \mathrm{q} 11.23$ including HIP1 have been identified in several patients $^{17-19}$. Based on genotype-phenotype correlation studies for such cases, HIP1 has been considered a candidate gene for neurological impairments. This is also supported by several functional studies ${ }^{20,21}$. The de novo heterozygous HIP1 variant identified in this study may suggest the previously considered hypothesis that HIP 1 is responsible for neurological impairments, although no marked protein conformational change was identified with a prediction tool.

In the present sibling cases, a previously reported $M E C P 2$ (the methyl CpG binding protein 2 gene) variant, p.Arg167Trp, was commonly observed as an X-linked recessive trait. This variant was previously identified in a three-generation family consisting of four individuals with non-specific X-linked $\mathrm{ID}^{22}$. Thus, p.Arg167Trp may have some clinical impact on the present sibling cases; however, patient 1 did not show developmental delay in the early stage. Thus, the severe neurological manifestations observed in patient 2 cannot be explained by the $M E C P 2$ p.Arg167Trp variant.

De novo variants are the major cause of neurodevelopmental disorders in childhood. In such cases, probands in their families are recognized as sporadic cases. Although de novo variants can be observed with an estimated background rate of 0.86 amino-acid-altering variants per newborn in controls ${ }^{23}$, it would be rare for de novo variants to have a pathogenic impact on the proteins related to important functions. Thus, consecutive occurrence of de novo variants in the same family is an extremely rare phenomenon. Only a few cases of such consecutive occurrence of de novo variants have been reported ${ }^{24}$. Here, we observed 


\section{different de novo variants in sibling cases with different phenotypic features.}

\section{HGV database}

The relevant data from this Data Report are hosted at the Human Genome Variation Database at: https://doi.org/10.6084/m9.figshare.hgv.2348; https:// doi.org/10.6084/m9.figshare.hgv.2351; https://doi.org/10.6084/m9.figshare. hgv. 2354

\section{Acknowledgements}

This study was supported in part by grant from Japan Agency of Medical Research and Development, AMED (Practical Research Project for Rare/Intractable Diseases: 17ek0109270) and Grants-in Aid from the Research Committee of Development of Systems for the Diagnosis, Treatment, and Study of Inherited White Matter Disorders, Health, Labour and Welfare Sciences Research Grants, the Ministry of Health, Labour and Welfare, Japan.

\section{Author details}

${ }^{1}$ Institute of Medical Genetics, Tokyo Women's Medical University, Tokyo, Japan. ${ }^{2}$ Tokyo Women's Medical University Institute for Integrated Medical Sciences, Tokyo, Japan. ${ }^{3}$ Epilepsy Centre, NHO Shizuoka Institute of Epilepsy and Neurological Disorders, Shizuoka, Japan. ${ }^{4}$ Department of Genetics, Yokohama City University, Yokohama, Japan

\section{Conflict of interest}

The authors declare that they have no conflict of interest.

\section{Publisher's note}

Springer Nature remains neutral with regard to jurisdictional claims in published maps and institutional affiliations.

Supplementary information is available for this paper at https://doi.org/ 10.1038/s41439-018-0020-z.

Received: 6 May 2018 Revised: 14 June 2018 Accepted: 22 June 2018. Published online: 19 July 2018

\section{References}

1. van der Knaap, M. S. \& Bugiani, M. Leukodystrophies: a proposed classification system based on pathological changes and pathogenetic mechanisms. Acta Neuropathol. 134, 351-382 (2017).

2. Barkovich, A. J. \& Deon, S. Hypomyelinating disorders: An MRI approach. Neurobiol. Dis. 87, 50-58 (2016).

3. Charzewska, A. et al. Hypomyelinating leukodystrophies-a molecular insight into the white matter pathology. Clin. Genet. 90, 293-304 (2016).

4. Ashrafi, M. R. \& Tavasoli, A. R. Childhood leukodystrophies: a literature review of updates on new definitions, classification, diagnostic approach and management. Brain. Dev. 39, 369-385 (2017).
5. Lu, Y., Ondo, Y., Shimojima, K, Osaka, H. \& Yamamoto, T. A novel TUBB4A mutation G96R identified in a patient with hypomyelinating leukodystrophy onset beyond adolescence. Hum. Genome Var. 4, 17035 (2017).

6. Magen, D. et al. Mitochondrial hsp60 chaperonopathy causes an autosomalrecessive neurodegenerative disorder linked to brain hypomyelination and leukodystrophy. Am. J. Hum. Genet. 83, 30-42 (2008).

7. Hansen, J. J. et al. Hereditary spastic paraplegia SPG13 is associated with a mutation in the gene encoding the mitochondrial chaperonin Hsp60. Am. J. Hum. Genet. 70, 1328-1332 (2002).

8. Yamamoto, T. \& Shimojima, K. Pelizaeus-Merzbacher disease as a chromosomal disorder. Congenit. Anom. (Kyoto). 53, 3-8 (2013).

9. Miyake, N. et al. Biallelic mutations in nuclear pore complex subunit NUP107 cause early-childhood-onset steroid-resistant nephrotic syndrome. Am. J. Hum. Genet. 97, 555-566 (2015).

10. Higasa, $\mathrm{K}$. et al. Human genetic variation database, a reference database of genetic variations in the Japanese population. J. Hum. Genet. 61, 547 (2016).

11. Miyatake, $\mathrm{S}$. et al. Detecting copy-number variations in whole-exome sequencing data using the eXome Hidden Markov Model: an 'exome-first' approach. J. Hum. Genet. 60, 175-182 (2015).

12. Abecasis, G. R. et al. An integrated map of genetic variation from 1,092 human genomes. Nature 491, 56-65 (2012).

13. Yamaguchi-Kabata, Y. et al. iJGVD: an integrative Japanese genome variation database based on whole-genome sequencing. Hum. Genome Var. 2, 15050 (2015).

14. Lopez-Hernandez, T. et al. Mutant GlialCAM causes megalencephalic leukoencephalopathy with subcortical cysts, benign familial macrocephaly, and macrocephaly with retardation and autism. Am. J. Hum. Genet. 88, 422-432 (2011).

15. Hansen, J. et al. A novel mutation in the HSPD1 gene in a patient with hereditary spastic paraplegia. J. Neurol. 254, 897-900 (2007).

16. Hewamadduma, C. A. et al. HSP60 is a rare cause of hereditary spastic paraparesis, but may act as a genetic modifier. Neurology 70, 1717-1718 (2008).

17. Fusco, C. et al. Smaller and larger deletions of the Williams Beuren syndrome region implicate genes involved in mild facial phenotype, epilepsy and autistic traits. Eur. J. Hum. Genet. 22, 64-70 (2014).

18. Komoike, Y. et al. Zebrafish gene knockdowns imply roles for human XWHAG in infantile spasms and cardiomegaly. Genesis 48, 233-243 (2010).

19. Ramocki, M. B. et al. Recurrent distal 7q11.23 deletion including HIP1 and YWHAG identified in patients with intellectual disabilities, epilepsy, and neurobehavioral problems. Am. J. Hum. Genet. 87, 857-865 (2010).

20. Peng, L. et al. Huntingtin-interacting protein 1-related protein plays a critical role in dendritic development and excitatory synapse formation in hippocampal neurons. Front. Mol. Neurosci. 10, 186 (2017)

21. Metzler, M. et al. Disruption of the endocytic protein HIP1 results in neurological deficits and decreased AMPA receptor trafficking. EMBO J. 22 3254-3266 (2003).

22. Couvert, P. et al. MECP2 is highly mutated in X-linked mental retardation. Hum Mol. Genet. 10, 941-946 (2001).

23. Vissers, L. E. et al. A de novo paradigm for mental retardation. Nat. Genet. 42 1109-1112 (2010).

24. Pasmant, $\mathrm{E}$. et al. Two independent de novo mutations as a cause for neurofibromatosis type 1 and Noonan syndrome in a single family. Am. J. Med. Genet. A. 158A, 2290-2291 (2012). 